\title{
Correction to: Galectin-3 and soluble RAGE as new biomarkers of post-infarction cardiac remodeling
}

\author{
Alfredo Redondo ${ }^{1} \cdot$ Beatriz Paradela-Dobarro ${ }^{2,3} \cdot$ Isabel Moscoso ${ }^{2,3} \cdot$ María Moure-Álvarez $^{1} \cdot$ María Cebro-Márquez $^{2}$. \\ José Ramón González-Juanatey ${ }^{1,2,3} \cdot$ Javier García-Seara ${ }^{1,2,3} \cdot$ Ezequiel Álvarez $^{3,4}$ (I)
}

Published online: 13 March 2021

(C) Springer-Verlag GmbH Germany, part of Springer Nature 2021

\section{Correction to: Journal of Molecular Medicine} https://doi.org/10.1007/s00109-021-02054-6

The original publication contains an error. Correct figure 1 is presented in this paper.

The original article has been corrected.

The online version of the original article can be found at https://doi.org/ 10.1007/s00109-021-02054-6

Ezequiel Álvarez

ezequiel.alvarez.castro@gmail.com

1 Servicio de Cardiología y Unidad de Hemodinámica, Complexo Hospitalario Universitario de Santiago de Compostela (CHUS), SERGAS, Travesía da Choupana s/n, Santiago de Compostela, 15706 A Coruña, Spain

2 Instituto de Investigación Sanitaria de Santiago de Compostela (IDIS), Complexo Hospitalario Universitario de Santiago de Compostela (CHUS), SERGAS, Travesía da Choupana s/n, Santiago de Compostela, 15706 A Coruña, Spain

3 CIBERCV, Madrid, Spain

4 Laboratorio No. 6. Edif. Consultas Externas (Planta-2), Instituto de Investigación Sanitaria de Santiago de Compostela (IDIS),

Complexo Hospitalario Universitario de Santiago de Compostela (CHUS), SERGAS, Travesía da Choupana s/n, Santiago de Compostela, 15706 A Coruña, Spain 

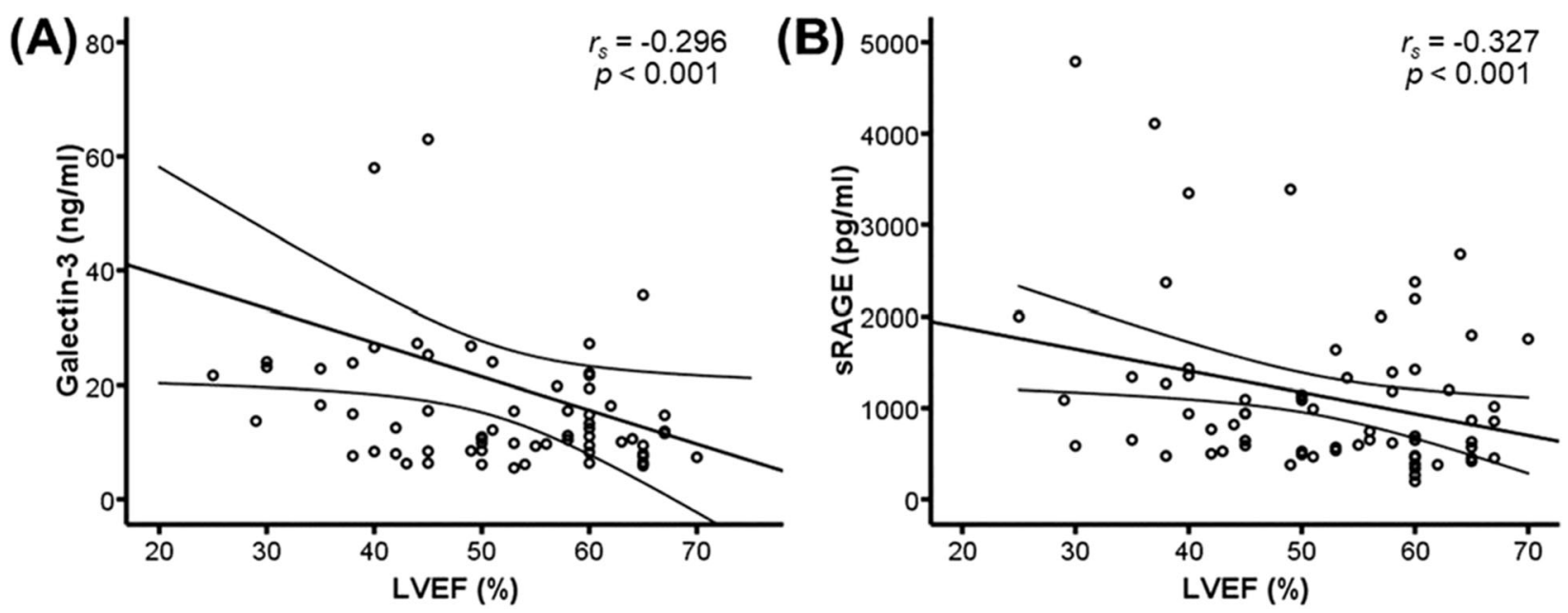

956 Fig. 1 Linear correlation between plasma levels during $48 \mathrm{~h}$ after STEMI of galectin-3 (a) and SRAGE (b) with the percentage of left ventricle ejection fraction (LVEF). The straight lines show total adjustment with their confidence intervals (curved lines). $r_{\mathrm{s}}=$ Spearman's correlation coefficient

Publisher's note Springer Nature remains neutral with regard to jurisdictional claims in published maps and institutional affiliations. 